\title{
ACCURACY OF MELD-NA SCORE AND OTHER NON-INVASIVE PREDICTORS OF ESOPHAGEAL VARICES STATUS IN EGYPTIAN PATIENTS WITH LIVER CIRRHOSIS
}

\author{
By \\ RASHA SAMIR MOHAMED ${ }^{1}$, MOHAMED LOTFY SOLIMAN ${ }^{1}$, \\ WEAL S. ELGHARABAWY ${ }^{2}$ and MANAL SABRY MOHAMED ${ }^{1}$ \\ Department of Internal Medicine ${ }^{1}$ and Department of Anesthesia ${ }^{2}$, Faculty of \\ Medicine, Ain Shams University, Cairo 11566, Egypt \\ ( ${ }^{\star}$ Correspondence: drousha1981@gmail.com) \\ Abstract
}

Esophageal varices (OV) are a serious and common complication of portal hypertension associated with liver cirrhosis. Hemorrhage from OV rupture is a life-threatening complication with high mortality. The model for end stage liver disease (MELD), MELD-Na+ scores are predictors of non-transplant surgical mortality among patients with cirrhosis and has been a useful tool predicting the mortality in patients awaiting liver transplantation. As well, they have been suggested in many studies as useful predictors of OV presence and severity.

This study evaluated accurate cut-off values of MELD-Na and aspartate aminotransferase to platelet count ratio index (APRI), AST/ALT (AAR), creatinine (ASR/Cr) as well as AST/ Bilirubin (AST/Bil.) ratios as new non-invasive predictors for liver cirrhosis patients.

Keywords: Patients, esophageal varices, MELD score, APRI, AAR, Non-invasive predictors, platelet count

\section{Introduction}

Generally, liver fibrosis is the major consequence of chronic hepatitis $\mathrm{C}(\mathrm{CHC})$ and representing a major global health problem (Friedman, 2000). Hepatitis C virus (HCV) was a worldwide health problem and a leading cause of chronic liver disease (Ghany et al, 2009). It was estimated that, hepatitis $\mathrm{C}$ virus genotype 4 (HCV-4) was the cause of approximately $20 \%$ of 180 million cases of chronic hepatitis $\mathrm{C}$ in the world. HCV-4 infection was common in the Middle East and Africa, highest in Egypt at $>10 \%$ of the general population and China has the most people with $\mathrm{HCV}, 29.8$ million (Abd El Razek et al, 2014).

The largest HCV epidemic was found in Egypt, with an estimated national prevalence reported to be $14.7 \%$ (Cuadros et al, 2014). End stage of chronic HCV infection is cirrhosis, ultimately complicated by portal hypertension, an established contributing factor in evolution of a variety of cirrhosis complications as ascites, hepatic encephalopathy, and esophageal varices (Elalfy, et al, 2016). Patients, who survived the first episode of esophageal hemorrhage, had a risk of recurrent bleeding up to $60 \%$ with a mortality rate up to $33 \%$ (Bari and Garcia-Tsao, 2012). Noninvasive predictors were recommended as a useful way to screen for the presence and severity OV (Herwanto et al, 2018).

This study aimed to evaluate the MELD$\mathrm{Na}$ accurate cut-off values \& other non-invasive predictors as aspartate aminotransferase to platelet count ratio index (APRI), AST/ALT (AAR), AST/ Creatinine (ASR/ $\mathrm{Cr}$ ) and AST/Bilirubin(AST/Bil.) ratios as a new non-invasive predictor for presence of $\mathrm{OV}$ and degree in liver cirrhosis patients

\section{Subjects and Methods}

The study included 60 patients with liver cirrhosis of variable etiology admitted to Ain-Shams University Hospitals, Hepatology and Gastroenterology unit in the period from February 2019 to October 2019. All were dealt with according to the ethical guidelines of Ain-Shams University Hospitals and after having a written consent from all. Patients were divided into 2 groups: GI: 40 patients with liver cirrhosis \& esophageal varices diagnosed by upper GIT endoscopy as (patients) which were subdivided into 4 groups of 10 patients each according to the esophageal varices, grade (SG1,SG2, SG3 \& 
SG4). GII: 20 patients with liver cirrhosis without esophageal varices (G-0) endoscopy evident as (control group).

Both groups were subjected to the following: 1- Full history taking \& clinical examination. 2- Laboratory investigations including: 1- CBC. 2- Liver function tests: ALT, AST, total bilirubin, direct bilirubin, Prothrombin time (PT) \& international normalization ratio (INR). 3- Renal function tests: serum urea, serum creatinine, serum sodium $\&$ potassium.

4- Abdominal ultrasound. 5- Upper GIT endoscopy under Propofol ${ }^{\circledR}$ IV to diagnose esophageal varices presence $\&$ its grade acco rding to: Grade I: Small straight cords of varices confined to the lower third of esophagus, Grade 2: Moderate sized clubbed varices, with well- defined areas of normal mucosa between them, forming several distinct variceal cords and confined to lower half of the esophagus, Grade 3: Gross varices extended into the proximal half of esophagus, normal mucosa might not be visible in-between them unless esophagus was fully distended with air and Grade 4: Varices like grade 3 but with dilated capillaries on top or in-between them (Dagradi et al, 1966).

6- MELD score evaluation according to the following formula: $\mathrm{MELD}=3.78[\mathrm{Ln}$ serum bilirubin $(\mathrm{mg} / \mathrm{dL})]+11.2[\mathrm{Ln} \mathrm{INR}]+9.57[\mathrm{Ln}$ serum creatinine $(\mathrm{mg} / \mathrm{dL})]+6.43$ (Kamath and Kim, 2007). If the initial MELD scores were 12 or more, the score was adjusted by incorporating the serum sodium value according to The United Network for Organ Sharing (White et al, 2019). 7- MELD-Na equation= MELD +1.32x (137-Na)- [0.033 x MELDx (137-Na)]. After Kalra et al. (2016) the MELD was utilized log scale calculations with a value less than 1 was given a lower limit value of 1 to prevent generating a negative score. The lower limit of serum sodium (Na) was capped at $125 \mathrm{mmol} / 1$, and the upper limit was capped at $137 \mathrm{mmol} / 1$. The upper limit of serum creatinine was capped at 4; in addition, if the patient had dialysis at least twice in the past week, the value for serum creatinine would be adjusted to 4.0, with maximum MELD score of 40. 8-APRI Score (De Fr-anchis, 2010): APRI $=$ AST (IU/L)/AST upper normal limit (IU/L) x100/Platelet count $\left(10^{9} / \mathrm{L}\right)$ or $[(\mathrm{AST} / \mathrm{ULN}$ AST $\mathrm{x}$ 100)/ Platelets $\left.\left(10^{9} / \mathrm{L}\right)\right]$.

9- Calculation of other suggested non-invasive predictors: AST/ALT Ratio (AAR), AST/Creatinine Ratio (AST/Cr) and AST/ Bilirubin (AST/Bil.) ratio.

Statistical analysis: Data were analyzed using SPSS ver. $18 \mathrm{IBM}^{\circledR}$ incorporation. Numerical data were tested for normality with D'Agostino-Pearson test (Willemsen et al, 2019), presented as mean \pm SD. Categorical data were presented as number and percent of total. Comparative analysis of numerical data was done with ANOVA with post hoc Bonferroni test. Comparative analysis of categorical data was done with Chisquare $\left(\mathrm{X}^{2}\right)$, or Fisher-exact test due to distributed frequencies. Correlations were tested using Spearman rank correlation and interpreted as in table. Receiver-operating characteristic curve analysis examined predictive value of APRI, AAR, MELD-Na+, AST/ creatinine or AST/bilirubin, Area under ROC curve (AUC) was interpreted as in table.

\begin{tabular}{|l|l|}
\hline Correlation coefficient (Spearman rho) & Strength of correlation \\
\hline$<0.2$ & Very weak \\
\hline $0.2-0.39$ & Weak \\
\hline $0.4-0.59$ & Moderate \\
\hline $0.6-0.79$ & Strong \\
\hline $0.8-1.0$ & Very strong \\
\hline \begin{tabular}{|l|l|}
\hline Area under ROC curve (AUC) & Predictive value \\
\hline $0.9-1.0$ & Excellent \\
\hline $0.8-0.89$ & Good \\
\hline $0.7-0.79$ & Fair \\
\hline $0.6-0.69$ & Poor \\
\hline$<0.6$ & Fail \\
\hline
\end{tabular}
\end{tabular}


P-value $<0.05$ was considered significant; data were tabulated and graphically illustrated, and probability (P-value) less than
0.05 was considered significant and less than 0.01 was as highly significant and more than 0.05 was considered nonsignificant.

\section{Results}

The results were shown in tables $(1,2 \& 3)$ and figures $(1,2,3,4,5,6 \& 7)$

Table 1: Comparison between patient $\&$ control groups as regard demographic data

\begin{tabular}{|c|c|c|c|c|c|c|c|c|c|c|c|}
\hline Variants & \multicolumn{2}{|c|}{$\begin{array}{c}\text { OV Grade I } \\
(10)\end{array}$} & \multicolumn{2}{|c|}{$\begin{array}{c}\text { OV Grade II } \\
(10)\end{array}$} & \multicolumn{2}{|c|}{$\begin{array}{c}\text { OV Grade III } \\
(10)\end{array}$} & \multicolumn{2}{|c|}{$\begin{array}{c}\text { OV Grade IV } \\
(10)\end{array}$} & \multicolumn{2}{|c|}{$\begin{array}{c}\text { Control } \\
(20)\end{array}$} & \multirow[t]{2}{*}{$P$ value } \\
\hline & Mean & SD & Mean & SD & Mean & SD & Mean & $\mathrm{SD}$ & Mean & SD & \\
\hline Age (year5) & $48.10 \mathrm{a}$ & 6.59 & $52.80 \mathrm{a}$ & 6.58 & $50.70 \mathrm{a}$ & 4.11 & $48.70 \mathrm{a}$ & 7.44 & $48.95 \mathrm{a}$ & 5.38 & 0.384 \\
\hline Male & \multicolumn{2}{|c|}{$8(80 \%)$} & \multicolumn{2}{|c|}{$6(60 \%)$} & \multicolumn{2}{|c|}{$7(70 \%)$} & \multicolumn{2}{|c|}{$10(100 \%)$} & \multicolumn{2}{|c|}{$12(60 \%)$} & \multirow[b]{2}{*}{0.148} \\
\hline Female & $2(20 \%)$ & & $4(40 \%)$ & & $3(30 \%)$ & & 0 & & $8(40 \%)$ & & \\
\hline
\end{tabular}

There was a significant positive correlation between OV grade and each of APRI, AAR, MELD-NA and AST creatinine, but without significant correlation between OV grade and AST/Bilirubin (Figs.1, 2, 3, 4 \& $5)$.

Table 2: ROC curve analysis for discrimination between patients with or without OV by using predictors.

\begin{tabular}{|l|c|c|c|c|c|}
\hline Case to Control & \multicolumn{5}{|c|}{ Predictor } \\
\hline ROC metric & APRI & AAR & MELD-Na $^{+}$ & AST/Creatinine & AST/Bilirubin \\
\hline AUC & 0.953 & 0.722 & 0.826 & 0.726 & 0.537 \\
\hline P-value* & $<0.0001$ & 0.0024 & $<0.0001$ & 0.0004 & 0.6192 \\
\hline Cut-off criterion & $>0.42$ & $>1.087$ & $>10.8$ & $>40.0$ & $>35.8$ \\
\hline Sensitivity, \% & 85.0 & 80.0 & 92.5 & 52.5 & 37.5 \\
\hline Specificity, \% & 95.0 & 65.0 & 65.0 & 90.0 & 100.0 \\
\hline$+\mathrm{PV}, \%$ & 97.1 & 82.1 & 84.1 & 91.3 & 100.0 \\
\hline -PV, \% & 76.0 & 61.9 & 81.3 & 48.6 & 44.4 \\
\hline
\end{tabular}

AUC $=$ area under the ROC curve, $+\mathrm{PV}=$ positive predictive value, $-\mathrm{PV}=$ negative predictive value, ${ }^{*}$ DeLong method.

Table 3: ROC curve analysis between OV-G0-2 and G3-4 by using predictors.

\begin{tabular}{|l|c|c|c|c|c|}
\hline G0-2 / G3-4 & \multicolumn{5}{|c|}{ Predictor } \\
\hline ROC metric & APRI & AAR & MELD-Na & AST/Creatinine & AST/Bilirubin \\
\hline AUC & 0.946 & 0.744 & 0.805 & 0.829 & 0.534 \\
\hline P-value* & $<0.0001$ & 0.0002 & $<0.0001$ & $<0.0001$ & 0.7279 \\
\hline Cut-off criterion & $>1.24$ & $>1.455$ & $>15.7$ & $>40.0$ & $\leq 12.5$ \\
\hline Sensitivity, \% & 80.0 & 70.0 & 55.0 & 80.0 & 30.0 \\
\hline Specificity, \% & 100.0 & 75.0 & 92.5 & 82.5 & 97.5 \\
\hline+ PV, \% & 100.0 & 58.3 & 78.6 & 69.6 & 85.7 \\
\hline -PV, \% & 90.9 & 83.3 & 80.4 & 89.2 & 73.6 \\
\hline
\end{tabular}

\section{Discussion}

Esophageal varices $(\mathrm{OV})$ being one of the commonest complications of liver cirrhosis responsible for a high mortality rate, while initiation of non- cardio- selective $\beta$ - blockers enabled a $50 \%$ reduction in the incidence of the first hemorrhage. Therefore, endoscopic screening for $\mathrm{OV}$ at time of diagnosis is strongly recommended by all clinical guidelines (Lin et al, 2011).

As an invasive technique endoscopy is not easily accepted by many patients raising the need for non- invasive approaches for $\mathrm{OV}$ prediction and identified patients who could benefit from non-selective beta-blockers therapy or start endoscopic prophylaxis (Zaman et al, 2001). The Child-Pugh and MELD scores had similar capability for predicting in-hospital overall mortality in patients with chronic liver disease. Besides, MELD was significantly better than Child-Pugh score for predicting hospital mortality due to variceal bleeding (Flores-Rendón et al, 2008).

The present study assessed different laboratory, parameters as a non-invasive methods for diagnosis and grading of esophageal varices in (60) patients with liver cirrhosis, without significant difference between their 
age $\&$ sex.

In the current study, MELD-Na score presented a good predictor of OV presence (AUC: 0.82 ) with sensitivity of $92.5 \%$ and specificity of $65 \%$ at cut off value $>10.8$, which agreed with a previous study that found MELD-Na at cut-off value of 11.5 had highest sensitivity (93.3\%) and specificity of $76 \%$ and it confirmed a positive correlation of MELD-Na with varices' grade (Ashraf and El-Sayed, 2018). The present study however showed MELD-Na score could discriminate bet-ween OV G0-2 \& G3-4 (AUC:0.80) with sensitivity of 55\% \& specificity of $92.5 \%$ at cut-off value $>15.7$. APRI score in this study was an excellent predictor of OV presence (AUC: 0.95) with sensitivity of $85 \%$ and specificity of $95 \%$ at cut off value $>0.42$. It had a positive correlation with esophageal varices grade with sensitivity $80 \%$ and specificity $100 \%$, at cutoff value $>1.24$. However, APRI at cutoff value showed 0.908 of $87.3 \%$ sensitivity and 71.4\% specificity (Mandal et al, 2019). Thus, AAR and AST/Cr ratios were fair predictors of OV presence with AUC:0.7 for each with sensitivity of $80 \%$ \& $52.5 \%$ and specificity of $65 \% \& 90 \%$ at cut off value $>1.087,>40.0$ respectively and both discriminated between OV Grades

In the present study, in the G0-2a \& G3-4, AAR was fair predictor for discrimination (AUC:0.72) with sensitivity of $70 \%$ and specificity of $75 \%$ at cut of values $>1.45$ and AST/Cr ratio a good predictor (AUC:0.85) with sensitivity of $80 \%$ and specificity of $82.5 \%$ at cut of values $>40$, these results goes partially with a study done in 2015 which concluded that APRI, AAR had modest diagnostic accuracy for varices in liver cirrhosis (Deng et al, 2015). However, in our study APRI score was excellent predictor, the difference in cut off values and sensitivity of predictors between the present study and the other studies may be due to racial and genetic differences. As for the knowledge, AST/Cr ratio as a predictor was not studied or suggested before.

\section{Conclusion}

Non-invasive predictors specially the APRI score among others (MELD Na score, and AAR as well as AST/Cr ratio) could be used as a non-invasive low cost screening of the esophageal varices presence and might even predict its grade as a trial to avoid unnecessary costly upper endoscopy in liver cirrhosis patients.

\section{References}

Abd El Razek, MA, Mahfouz, H, Afify, M, et al, 2014: Detection of risky esophageal varices by $2 \mathrm{D}$ U/S: When to perform endoscopy? Am. J. Med. Sci. 347:28-33.

Ashraf, DG, El-Sayed, I, 2018: Esophageal varices predictive score in liver cirrhosis. Egypt. J. Inter. Med. 30, 2:72-7.

Bari, K, Garcia-Tsao, G, 2012: Treatment of portal hypertension. World J. Gastroenterol. 18, 11:1166-75.

Burton, JR, Liangpunsakul, S, Lapidus, J, Giannini, E, et al, 2007: Validation of a multivariate model predicting presence and size of varices. J. Clin. Gastroenterol. 41, 6:609-15.

Cuadros, DF, Branscum, AJ, Miller, FD, Abu-Raddad, LJ, 2014: Spatial epidemiology of hepatitis C virus infection in Egypt: An-alyses and implications. Hepatology 60:1150-9

Dagradi, AE, Stempien, SJ, Owens, LK, 1966: Bleeding esophagogastric varices: An endoscopic study of 50 cases. Arch. Surg. 92:944-7

De Franchis, R, 2010: Revising consensus in portal hypertension: Report of the Baveno $\mathrm{V}$ consensus workshop on methodology of diagnosis and therapy in portal hypertension. J. Hepatol. 53:762-8.

Deng, H, Qi, X, Peng, Y, Li, J, Li, H, et al, 2015: Diagnostic accuracy of APRI, AAR, Fib$4, \mathrm{Fi}$, and king scores for diagnosis of esophageal varices in liver cirrhosis: A retrospective study. Med. Sci. Monit. 21:3961-77.

Elalfy, H, Elsherbiny, W, Abdel Rahman, A, Elhammady, D, Shaltout, SW, et al, 2016: Diagnostic non-invasive model of large risky esophageal varices in cirrhotic hepatitis CV patients. World J. Hepatol. 8, 24:1028-37.

Flores-Rendón, AR, González-González, J A, García-Compean, D, Maldonado-Garza, HJ, Garza-Galindo, AA, 2008: Model for end stage of liver disease (MELD) is better than the ChildPugh score for predicting in-hospital mortality 
related to esophageal variceal bleeding. Ann. Hepatol. 7, 3:230-4.

Friedman, SL, 2000: Molecular regulation of hepatic fibrosis, an integrated cellular response to tissue injury. J. Biol. Chem. 275:2247-50.

Herwanto, V, Wang, Y, Shojaei, M, Tang, B, McLean, AS, 2018: P001 Reduced cellular respiration and ATP production in an in vitro model of sepsis. Crit. Care 22, 1:S82-8.

Kalra, A, Wedd, JP, Biggins, SW, 2016: Changing prioritization for transplantation: MELD$\mathrm{Na}$, hepatocellular carcinoma exceptions, and more. Curr. Opin. Organ. Transpla- nt. 21:120-6 Kamath, PS, Kim, WR, 2007: Advanced liver disease study group: The model for end-stage liver disease (MELD). Hepatology 45: 797-805

Lin, ZH, Xin, YN, Dong, QJ, et al, 2011: Performance of the aspartate aminotransferaseto-platelet ratio index for the staging of hepatitis C-related fibrosis: an updated meta-analysis. Hepatology 53:726-36
Mandal, AK, Subedi, P, Sharma, M, Suman, A, Thapa, S, et al, 2019: Prediction of aspartate aminotransferase to platelet ratio index with size of esophageal varices in liver cirrhosis. J. Nepal Hlth. Res. Counc.17, 42:38-41.

White, SL, Rawlinson, W, Boan, P, Sheppeard, V, Wong, G, et al, 2019: Infectious disease transmission in solid organ transplantation: Donor evaluation, recipient risk, and outcomes of transmission. Transplant. Direct. Jan; 5(1): e416. Published online 2018 Dec 20.

Willemsen, L, Neele, AE, Velden, SV, Prange, KHM, den Toom, M, et al, 2919: Peritoneal macrophages have an impaired immune response in obesity which can be reversed by subsequent weight loss. BMJ Open Diabet. Res. Care 7, 1:e000751.

Zaman, A, Beker, T, Lapido, J, et al, 2001: Risk factors for the presence of varices in cirrhotic patients without a history of variceal hemorrhage. Arch. Intern. Med. 161:2564-70

\section{Explanation of figures}

Figs.1- 5: Box plot illustrating the correlation between OV grade and APRI score (Fig. 1), AAR (Fig. 2), MELD-Na ${ }^{+}$(Fig. 3), AST/creatinine ratio (Fig. 4), AST/bilirubin ratio (Fig. 5). Box represents the interquartile range. Line inside box represented median. Whiskers represent minimum and maximum values. Dots represent individual observation)

Fig.6: Receiver-operating characteristic (ROC) curves for discrimination between patients with or without esophageal varices using APRI, AAR, MELD-Na+, AST/creatinine ratio or AST/bilirubin ratio.

Fig.7: Receiver-operating characteristic (ROC) curves for discrimination between patients with grade 3-4 or grade 0-2 esophageal varices using APRI, AAR, MELD-Na+, AST/creatinine or AST/bilirubin.

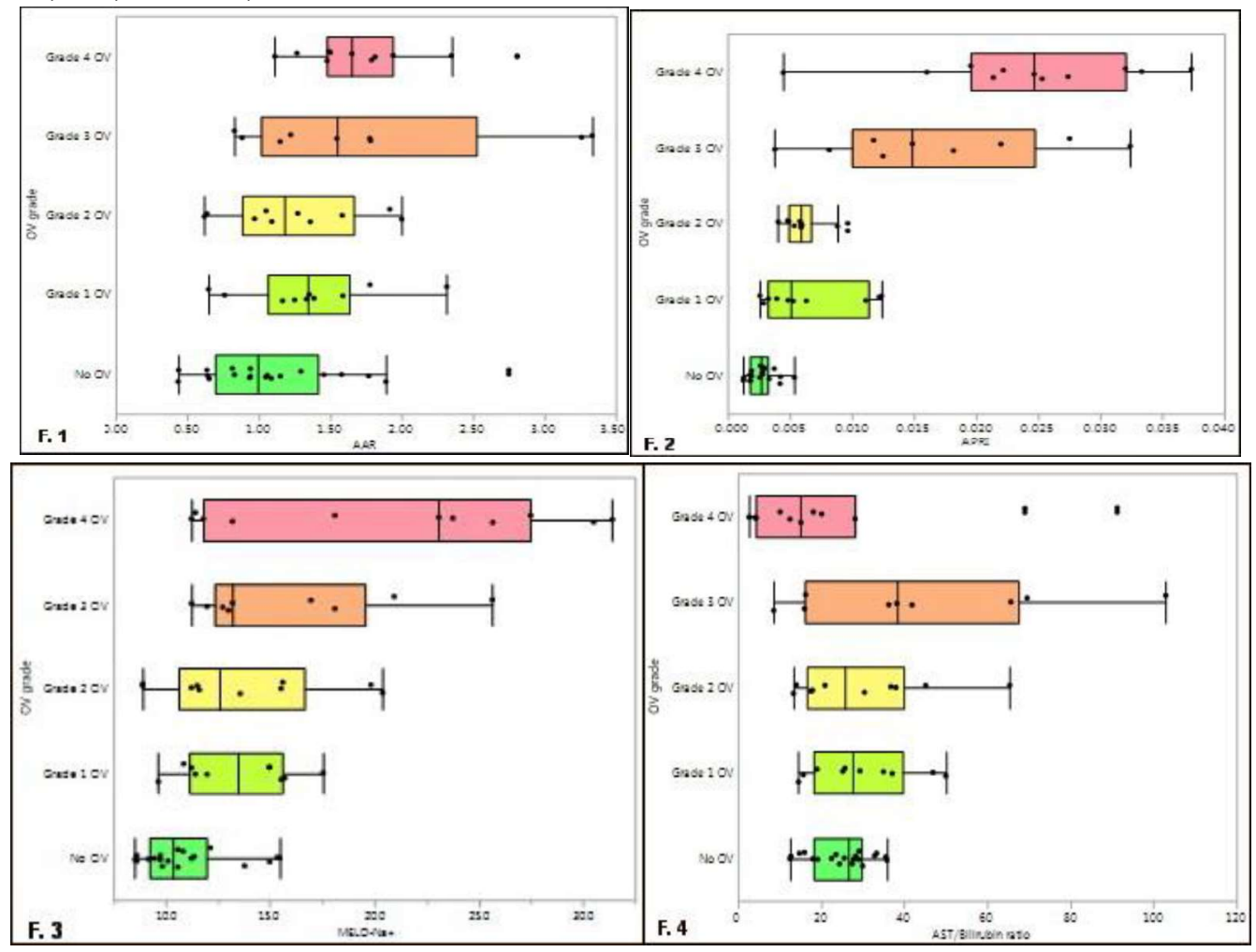



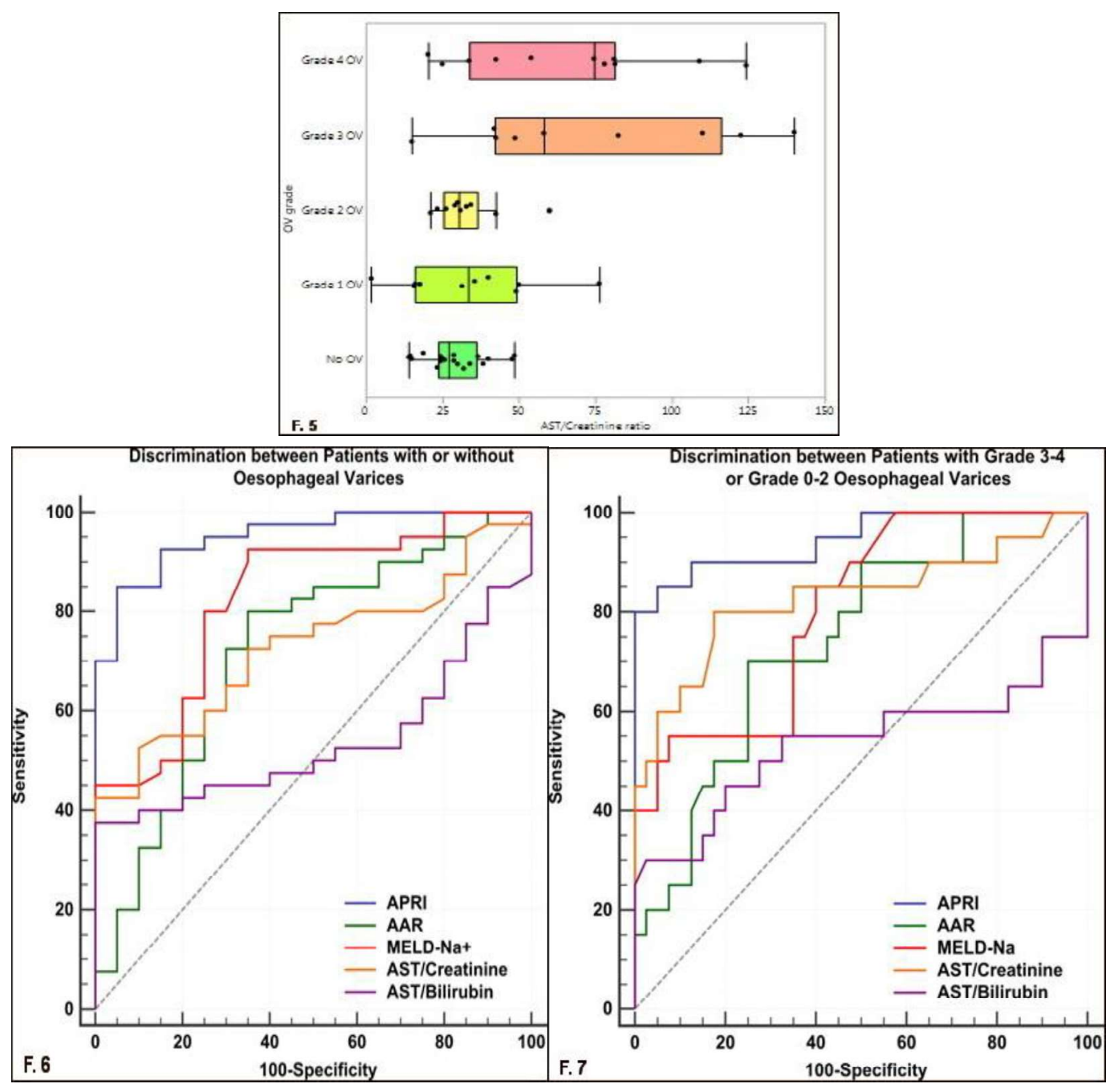